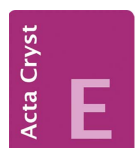

CRYSTALLOGRAPHIC COMMUNICATIONS

ISSN 2056-9890

Received 14 July 2016

Accepted 1 August 2016

Edited by I. D. Brown, McMaster University, Canada

Keywords: crystal structure; $\mathrm{CuMg}_{2}$ structure type; titanium; bismuth.

CCDC reference: 1497032

Supporting information: this article has supporting information at journals.iucr.org/e

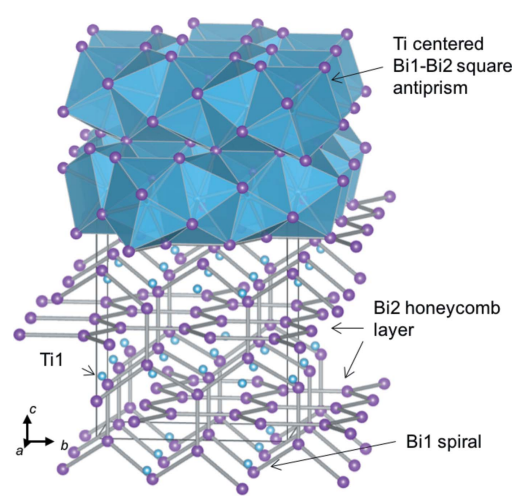

OPEN $\odot$ ACCESS

\section{Crystal structure of $\mathrm{TiBi}_{2}$}

\author{
Kei Watanabe and Hisanori Yamane*
}

Institute of Multidisciplinary Research for Advanced Materials, Tohoku University, 2-1-1 Katahira, Aoba-ku, Sendai 9808577, Japan. *Correspondence e-mail: yamane@tagen.tohoku.ac.jp

Black granular single crystals of monotitanium dibismuth, $\mathrm{TiBi}_{2}$, were synthesized by slow cooling of a mixture of $\mathrm{Bi}$ and $\mathrm{Ti}$ from $693 \mathrm{~K}$. The title compound is isostructural with $\mathrm{CuMg}_{2}$ (orthorhombic Fddd symmetry). Ti atoms are located in square antiprisms of $\mathrm{Bi}$ atoms. The network of one type of $\mathrm{Bi}$ atom spirals along the $a$-axis direction while honeycomb layers of the other type of $\mathrm{Bi}$ atom spreading in the $a b$ plane interlace one another.

\section{Chemical context}

$\mathrm{TiBi}_{2}$ was first reported in the study of the Ti-Bi binary phase diagram by Vassilev (2006). Maruyama et al. (2013) confirmed the presence of $\mathrm{TiBi}_{2}$ in their phase-diagram study and showed that the powder X-ray diffraction (XRD) pattern was consistent with that of a Ti-Bi film prepared by RF sputtering (Simić \& Marinković, 1990). However, the crystal system, lattice parameters and structure of $\mathrm{TiBi}_{2}$ were not reported.

In the present study, we prepared single crystals of $\mathrm{TiBi}_{2}$ to clarify the structure. The pellet of the starting mixture maintained the original shape after heating at $693 \mathrm{~K}$. The powder XRD pattern of the sample showed that a mixture of $\mathrm{TiBi}_{2}, \mathrm{Bi}$, and $\mathrm{Ti}$ had been obtained. Single crystals of $\mathrm{TiBi}_{2}$ approximately $120 \mu \mathrm{m}$ in size were picked up from the fractured sample. $\mathrm{TiBi}_{2}$ is unstable and decomposes in air. When the mixture was heated at $703 \mathrm{~K}$, the obtained sample was a mixture of $\mathrm{Bi}$ and $\mathrm{Ti}_{8} \mathrm{Bi}_{9}$. This temperature was above the peritectic temperature of $\mathrm{TiBi}_{2}(698 \mathrm{~K})$ reported in the phase diagram by Maruyama et al. (2013).

\section{Structural commentary}

$\mathrm{TiBi}_{2}$ is isotypic with $\mathrm{CuMg}_{2}$ (Schubert \& Anderko, 1951; Gingl et al., 1993), $\mathrm{NbSn}_{2}, \mathrm{VSn}_{2}, \mathrm{CrSn}_{2}$ (Wölpl \& Jeitschko, 1994; Larsson \& Lidin, 1995), and $\operatorname{IrIn}_{2}$ (Zumdick et al., 2000). $\mathrm{TiSnSb}$ is the only reported compound which contains $\mathrm{Ti}$ and crystallizes in the $\mathrm{CuMg}_{2}$-type structure (Malaman \& Steinmetz, 1979; Dashjav \& Kleinke, 2003). The crystal structure of $\mathrm{TiSb}_{2}$ adopts the $\mathrm{CuAl}_{2}$ type, while that of $\mathrm{TiSn}_{2}$ is not known. $\mathrm{TiBi}_{2}$ is the first binary compound that is composed of Ti and a group 15 element and has the $\mathrm{CuMg}_{2}$-type structure.

Fig. 1 shows the crystal structure of $\mathrm{TiBi}_{2}$ while the coordination environments of the Ti1, Bi1, and $\mathrm{Bi} 2$ atoms are illustrated in Fig. 2. The Ti1 site is located in a square antiprism of $\mathrm{Bi}$ atoms. The $\mathrm{Bi}$ square antiprisms are aligned alternately along the $a+b$ and $a-b$ directions by sharing the square planes. $\mathrm{Bi}-\mathrm{Ti}$ bond lengths in the $\mathrm{Bi}$ square antiprism and the $\mathrm{Ti}-\mathrm{Ti}$ distance of the inter-antiprisms are 2.9382 (16)3.0825 (6) and 2.9546 (2) $\AA$, respectively, which are in the 


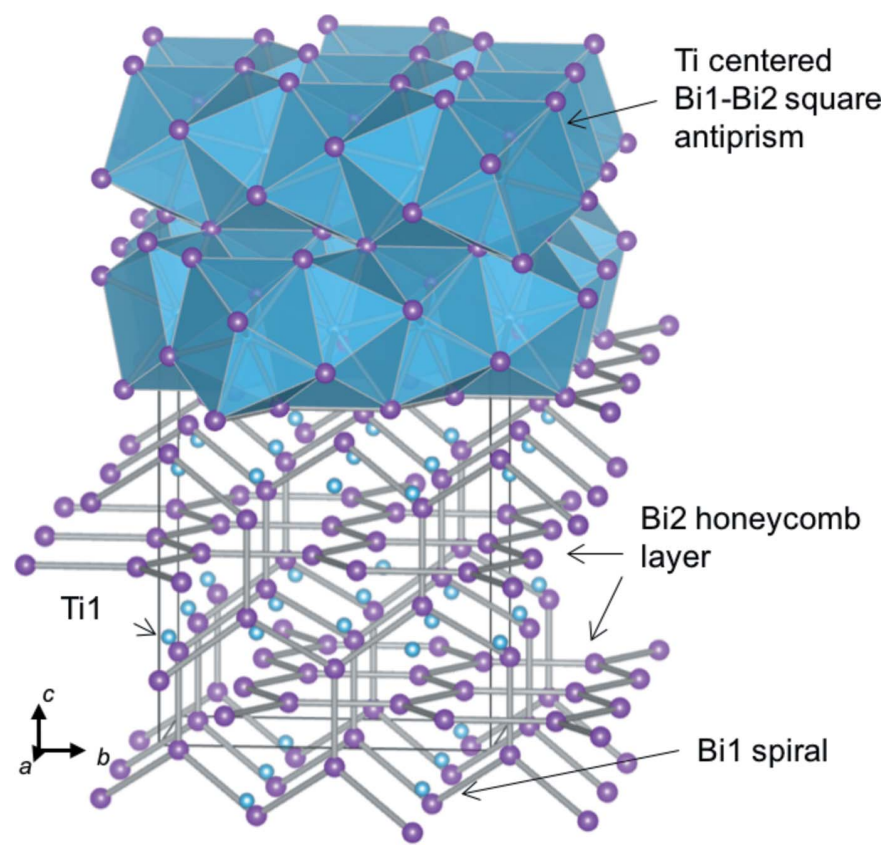

Figure 1

Crystal structure of $\mathrm{TiBi}_{2}$ illustrated with Ti-centered $\mathrm{Bi} 1-\mathrm{Bi} 2$ square antiprisms and $\mathrm{Bi} 1-\mathrm{Bi} 1$ and $\mathrm{Bi} 2-\mathrm{Bi} 2$ bonds.

ranges reported for $\mathrm{Ti}_{8} \mathrm{Bi}_{9}[\mathrm{Bi}-\mathrm{Ti}=2.818$ (4)-3.144 (6) $\AA$ and $\mathrm{Ti}-\mathrm{Ti}=2.934$ (6)-3.715 (5) Å; Richter \& Jeitschko, 1997].

The Bi1-Bi1 bond lengths in the Bi1 spiral-like network are 3.0730 (8) $\AA$ in the $c$-axis direction and 3.4589 (4) $\AA$ in the other direction. The $\mathrm{Bi} 2-\mathrm{Bi} 2$ bond lengths in the $\mathrm{Bi} 2$ honeycomb layers in the $a b$ plane are $3.4639(8) \AA$ in the $b$-axis direction and 3.3435 (4) $\AA$ in the other direction. The $\mathrm{Bi}-\mathrm{Bi}$ bond lengths in the spiral rings and honeycomb layers in $\mathrm{TiBi}_{2}$ are in the range of those in $\mathrm{Bi}$ metal (3.071 and $3.529 \AA$ A Cucka \& Barrett, 1962). The interatomic distances between the $\mathrm{Bi}$ atoms of the spiral network and the honey-

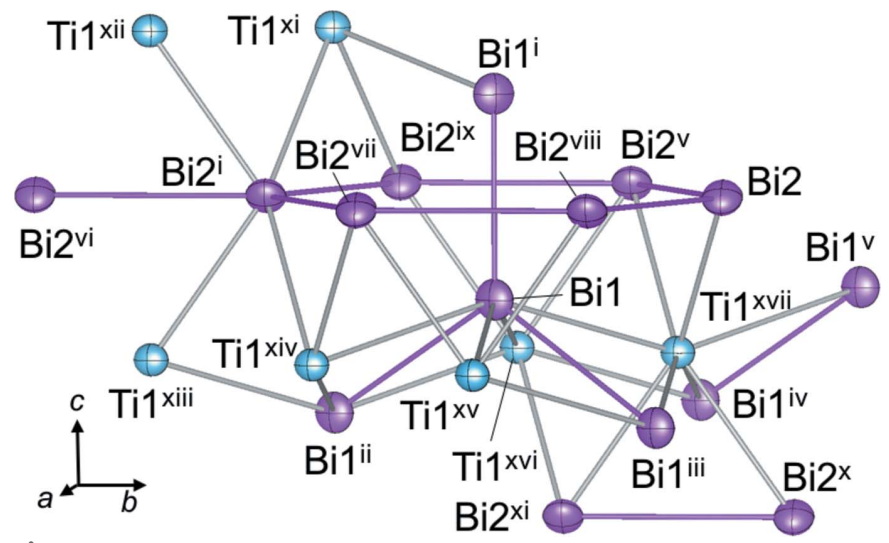

Figure 2

The atomic arrangement around $\mathrm{Ti}$ and $\mathrm{Bi}$ atoms in the structure of $\mathrm{TiBi}_{2}$. Displacement ellipsoids are drawn at $99 \%$ probability. [Symmetry codes: (i) $x,-y+\frac{1}{4},-z+\frac{1}{4}$; (ii) $-x,-y,-z$; (iii) $x+\frac{1}{4}, y+\frac{1}{4},-z$; (iv) $x-\frac{3}{4}, y+\frac{1}{4}$, $-z$; (v) $-x-\frac{1}{4},-y+\frac{3}{4}, z$; (vi) $x, y-1, z$; (vii) $-x+\frac{3}{4}, y-\frac{1}{2},-z+\frac{1}{4}$; (viii) $-x+\frac{3}{4},-y+\frac{3}{4}, z$; (ix) $-x-\frac{1}{4}, y-\frac{1}{2},-z+\frac{1}{4} ;(x)-x,-y+1,-z$; (xi) $-x$, $y-\frac{1}{4}, z-\frac{1}{4}$; (xii) $x,-y-\frac{1}{4},-z+\frac{3}{4}$; (xiii) $x, y-\frac{1}{2}, z-\frac{1}{2}$; (xiv) $x+\frac{1}{4}, y-\frac{1}{4},-$ $z+\frac{1}{2} ;(\mathrm{xv})-x+\frac{3}{4},-y+\frac{1}{4}, z-\frac{1}{2} ;(\mathrm{xvi})-x-\frac{1}{4},-y+\frac{1}{4}, z-\frac{1}{2} ;(\mathrm{xvii})-x,-y+\frac{1}{2}$, $-z+\frac{1}{2}$.]
Table 1

Experimental details.

Crystal data

Chemical formula

$M_{\mathrm{r}}$

Crystal system, space group

Temperature (K)

$a, b, c(\AA)$

$V\left(\AA^{3}\right)$

$Z$

Radiation type

$\mu\left(\mathrm{mm}^{-1}\right)$

Crystal size (mm)

$\mathrm{TiBi}_{2}$

465.86

Orthorhombic, Fddd

298

5.7654 (4), $10.3155(6)$

$19.4879(12)$

$1159.00(13)$

16

Mo $K \alpha$

123.50

$0.14 \times 0.09 \times 0.06$

Data collection

Diffractometer

Absorption correction

$T_{\min }, T_{\max }$

No. of measured, independent and observed $[I>2 \sigma(I)]$ reflections

$R_{\text {int }}$

$(\sin \theta / \lambda)_{\max }\left(\AA^{-1}\right)$

Bruker D8 goniometer

Numerical ( $S A D A B S$; Bruker, 2014)

$0.016,0.102$

3881, 339, 309

0.048

0.649

Refinement

$R\left[F^{2}>2 \sigma\left(F^{2}\right)\right], w R\left(F^{2}\right), S$

No. of reflections

No. of parameters

$0.024,0.062,1.31$

339

17

$\Delta \rho_{\max }, \Delta \rho_{\min }\left(\mathrm{e} \AA^{-3}\right)$

$2.54,-3.80$

Computer programs: Instrument Service, APEX2 and SAINT-Plus (Bruker, 2014), SHELXL2014 (Sheldrick, 2015) and VESTA (Momma \& Izumi, 2011).

comb layers (Bi1-Bi2) are $3.6974(3), 3.7309(4)$ and 3.7546 (4) $\AA$, which are longer than the $\mathrm{Bi}-\mathrm{Bi}$ bond lengths in Bi metal.

\section{Synthesis and crystallization}

Starting powders of Bi (1 mmol, Mitsuwa Chemicals Co., Ltd, 99.999\%) and $\mathrm{Ti}(0.5 \mathrm{mmol}$, Mitsuwa Chemicals Co., Ltd, $99.99 \%$ ) were weighed, mixed in an alumina mortar with a pestle and formed into a pellet by uniaxial pressing in an $\mathrm{Ar}$ gas-filled glove box $\left(\mathrm{O}_{2}\right.$ and $\mathrm{H}_{2} \mathrm{O}<1$ p.p.m.). The pellet was put in a tantalum boat (Nilaco Corp., 99.95\%). The boat was sealed in a stainless-steel (SUS 316) tube. The sample was heated to $693 \mathrm{~K}$ in an electric furnace with a heating rate of $3.5 \mathrm{~K} \mathrm{~min}^{-1}$. This temperature was kept for $10 \mathrm{~h}$, and then lowered to $473 \mathrm{~K}$ with a cooling rate of $5 \mathrm{~K} \mathrm{~h}^{-1}$. After cooling to room temperature by shutting off the electrical power to the furnace, the stainless-steel tube was cut and opened in the glove box. To identify the crystalline phases, powder XRD $(\mathrm{Cu}$ $K \alpha$, Bruker, D2 phaser) was carried out for a portion of the sample which was ground in the alumina mortar and sealed under an Ar atmosphere in a holder with a kapton film window. The chemical compositions of $\mathrm{TiBi}_{2}$ single crystals placed on a carbon tape were determined with an electron probe microanalyzer (EPMA, JEOL, JXA-8200). $\mathrm{Bi}$ and $\mathrm{TiO}_{2}$ (Japan Electronics Co., Ltd) were used as standard samples. The analyzed composition ratio of $\mathrm{Ti}: \mathrm{Bi}$ in the crystals was 1.0 (1):2.0 (1). A single crystal of $\mathrm{TiBi}_{2}$ was sealed in a glass 
capillary with Ar gas in the glove box for the single-crystal XRD experiment.

\section{Refinement}

Crystal data, data collection and structure refinement details are summarized in Table 1.

\section{Acknowledgements}

This work was supported in part by a Grant-in-Aid for Scientific Research (B) (No. 16H04494) from the Ministry of Education, Culture, Sports and Technology (MEXT), Japan.

\section{References}

Bruker (2014). Instrument Service, APEX2, SADABS and SAINTPlus. Bruker AXS Inc., Madison, Wisconsin, USA.

Cucka, P. \& Barrett, C. S. (1962). Acta Cryst. 15, 865-872.
Dashjav, E. \& Kleinke, H. (2003). J. Solid State Chem. 176, 329 337.

Gingl, F., Selvam, P. \& Yvon, K. (1993). Acta Cryst. B49, 201203.

Larsson, A. K. \& Lidin, S. (1995). J. Alloys Compd. 221, 136142.

Malaman, B. \& Steinmetz, J. (1979). J. Less-Common Met. 65, 285288.

Maruyama, S., Kado, Y. \& Uda, T. (2013). J. Phase Equilib. Diffus. 34, 289-296.

Momma, K. \& Izumi, F. (2011). J. Appl. Cryst. 44, 1272-1276.

Richter, C. G. \& Jeitschko, W. (1997). J. Solid State Chem. 134, 2630.

Schubert, K. \& Anderko, K. (1951). Z. Metallkd. 42, 321-325.

Sheldrick, G. M. (2015). Acta Cryst. C71, 3-8.

Simić, V. \& Marinković, Z. (1990). Thin Solid Films, 191, 165-171.

Vassilev, G. P. (2006). Cryst. Res. Technol. 41, 349-357.

Wölpl, T. \& Jeitschko, W. (1994). J. Alloys Compd. 210, 185-190.

Zumdick, M. F., Landrum, G. A., Dronskowski, R., Hoffmann, R. D. \& Pöttgen, R. (2000). J. Solid State Chem. 150, 19-30. 


\section{supporting information}

Acta Cryst. (2016). E72, 1254-1256 [https://doi.org/10.1107/S2056989016012391]

\section{Crystal structure of $\mathrm{TiBi}_{2}$}

\section{Kei Watanabe and Hisanori Yamane}

\section{Computing details}

Data collection: Instrument Service (Bruker, 2014); cell refinement: APEX2 (Bruker, 2014); data reduction: SAINT-Plus (Bruker, 2014); program(s) used to solve structure: APEX2 (Bruker, 2014); program(s) used to refine structure: SHELXL2014 (Sheldrick, 2015); molecular graphics: VESTA (Momma \& Izumi, 2011).

Titanium dibismuth

\section{Crystal data}

$\mathrm{TiBi}_{2}$

$M_{r}=465.86$

Orthorhombic, Fddd

$a=5.7654$ (4) $\AA$

$b=10.3155$ (6) $\AA$

$c=19.4879(12) \AA$

$V=1159.00(13) \AA^{3}$

$Z=16$

$F(000)=3008$

Data collection

Bruker D8 goniometer diffractometer

Radiation source: micro focus sealed tube Detector resolution: 10.4167 pixels $\mathrm{mm}^{-1}$ $\omega, \varphi$ scans

Absorption correction: numerical (SADABS; Bruker, 2014)

$T_{\min }=0.016, T_{\max }=0.102$

Refinement

Refinement on $F^{2}$

Least-squares matrix: full

$R\left[F^{2}>2 \sigma\left(F^{2}\right)\right]=0.024$

$w R\left(F^{2}\right)=0.062$

$S=1.31$

339 reflections

17 parameters

0 restraints

Primary atom site location: dual
$D_{\mathrm{x}}=10.679 \mathrm{Mg} \mathrm{m}^{-3}$

Mo $K \alpha$ radiation, $\lambda=0.71073 \AA$

Cell parameters from 4528 reflections

$\theta=4.2-31.4^{\circ}$

$\mu=123.50 \mathrm{~mm}^{-1}$

$T=298 \mathrm{~K}$

Granule, black

$0.14 \times 0.09 \times 0.06 \mathrm{~mm}$

3881 measured reflections

339 independent reflections

309 reflections with $I>2 \sigma(I)$

$R_{\text {int }}=0.048$

$\theta_{\max }=27.5^{\circ}, \theta_{\min }=4.2^{\circ}$

$h=-7 \rightarrow 7$

$k=-13 \rightarrow 13$

$l=-25 \rightarrow 25$

Secondary atom site location: difference Fourier

$$
\text { map }
$$

$w=1 /\left[\sigma^{2}\left(F_{0}^{2}\right)+(0.0275 P)^{2}+47.1241 P\right]$

where $P=\left(F_{\mathrm{o}}^{2}+2 F_{\mathrm{c}}{ }^{2}\right) / 3$

$(\Delta / \sigma)_{\max }<0.001$

$\Delta \rho_{\max }=2.54 \mathrm{e} \AA^{-3}$

$\Delta \rho_{\min }=-3.80$ e $\AA^{-3}$

Extinction correction: SHELXL2014

(Sheldrick, 2015),

$\mathrm{Fc}^{*}=\mathrm{kFc}\left[1+0.001 \mathrm{xFc}^{2} \lambda^{3} / \sin (2 \theta)\right]^{-1 / 4}$

Extinction coefficient: 0.00038 (3) 


\section{Special details}

Geometry. All esds (except the esd in the dihedral angle between two 1.s. planes) are estimated using the full covariance matrix. The cell esds are taken into account individually in the estimation of esds in distances, angles and torsion angles; correlations between esds in cell parameters are only used when they are defined by crystal symmetry. An approximate (isotropic) treatment of cell esds is used for estimating esds involving l.s. planes.

Fractional atomic coordinates and isotropic or equivalent isotropic displacement parameters $\left(\AA^{2}\right)$

\begin{tabular}{lllll}
\hline & $x$ & $y$ & $z$ & $U_{\text {iso }} * / U_{\text {eq }}$ \\
\hline Bi1 & 0.1250 & 0.1250 & $0.04615(2)$ & $0.0064(2)$ \\
Bi2 & 0.1250 & $0.45710(4)$ & 0.1250 & $0.0066(2)$ \\
Ti1 & 0.1250 & 0.1250 & $0.49898(10)$ & $0.0050(4)$ \\
\hline
\end{tabular}

Atomic displacement parameters $\left(\mathscr{A}^{2}\right)$

\begin{tabular}{lllllll}
\hline & $U^{11}$ & $U^{22}$ & $U^{33}$ & $U^{12}$ & $U^{13}$ & $U^{23}$ \\
\hline Bi1 & $0.0035(3)$ & $0.0076(3)$ & $0.0082(3)$ & $0.00176(15)$ & 0.000 & 0.000 \\
Bi2 & $0.0058(3)$ & $0.0080(3)$ & $0.0060(3)$ & 0.000 & $-0.00212(15)$ & 0.000 \\
Ti1 & $0.0039(9)$ & $0.0060(9)$ & $0.0051(8)$ & $0.0005(8)$ & 0.000 & 0.000 \\
\hline
\end{tabular}

Geometric parameters $\left(\AA,{ }^{\circ}\right)$

\begin{tabular}{|c|c|c|c|}
\hline $\mathrm{Ti} 1-\mathrm{Bi} 2^{\mathrm{i}}$ & $2.9382(16)$ & $\mathrm{Bi} 1-\mathrm{Bi} 2^{\mathrm{xix}}$ & $3.7546(4)$ \\
\hline $\mathrm{Ti} 1-\mathrm{Bi} 2^{\mathrm{ii}}$ & $2.9382(16)$ & $\mathrm{Bi} 1-\mathrm{Bi} 2$ & $3.7546(4)$ \\
\hline $\mathrm{Ti} 1-\mathrm{Bi} 2^{\mathrm{iii}}$ & $3.0051(16)$ & Bi1-Ti1 ${ }^{\mathrm{xx}}$ & $3.0257(6)$ \\
\hline $\mathrm{Ti} 1-\mathrm{Bi}^{\mathrm{iv}}$ & $3.0051(16)$ & Bi1-Ti1 ${ }^{\mathrm{xxi}}$ & $3.0257(6)$ \\
\hline $\mathrm{Ti} 1-\mathrm{Bi}^{\mathrm{v}}$ & $3.0257(6)$ & Bi1-Til ${ }^{\text {vii }}$ & $3.0825(6)$ \\
\hline $\mathrm{Ti} 1-\mathrm{Bi}^{\mathrm{vi}}$ & $3.0257(6)$ & Bi1-Ti1 ${ }^{\mathrm{i}}$ & $3.0825(6)$ \\
\hline Ti1-Bi1 vii & $3.0825(6)$ & Bi1-Ti1 $1^{x x i i}$ & $4.9243(16)$ \\
\hline $\mathrm{Ti} 1-\mathrm{Bi}^{\mathrm{i}}$ & $3.0825(6)$ & Bi1-Ti1 ${ }^{\text {xiii }}$ & $4.9243(16)$ \\
\hline Ti1-Ti1 viii & $2.9546(2)$ & Bi1-Ti1 ${ }^{\text {xxiv }}$ & $4.9348(16)$ \\
\hline Ti1-Ti1 ${ }^{\text {ix }}$ & $2.9546(2)$ & Bil-Ti1 ${ }^{\mathrm{xxv}}$ & $4.9348(16)$ \\
\hline $\mathrm{Bi} 1-\mathrm{Bi} 1^{\mathrm{x}}$ & $3.0730(8)$ & Bi1-Ti1 ${ }^{\mathrm{xxvi}}$ & $5.1110(4)$ \\
\hline $\mathrm{Bi} 1-\mathrm{Bi}^{\mathrm{xi}}$ & $3.4589(4)$ & $\mathrm{Bi} 2-\mathrm{Ti}^{\mathrm{i}}$ & $2.9382(16)$ \\
\hline $\mathrm{Bi} 1-\mathrm{Bi1} 1^{\mathrm{xii}}$ & $3.4589(4)$ & Bi2-Ti1 ${ }^{\text {xiv }}$ & $2.9382(16)$ \\
\hline $\mathrm{Bi} 2-\mathrm{Bi} 2^{\mathrm{xiii}}$ & $3.3435(4)$ & $\mathrm{Bi} 2-\mathrm{Ti} 1^{\mathrm{xxvii}}$ & $3.0051(16)$ \\
\hline $\mathrm{Bi} 2-\mathrm{Bi} 2^{\mathrm{xiv}}$ & $3.3435(4)$ & Bi2-Ti1 $1^{\mathrm{xxviii}}$ & $3.0051(16)$ \\
\hline $\mathrm{Bi} 2-\mathrm{Bi}^{\mathrm{xv}}$ & $3.4639(8)$ & $\mathrm{Bi} 2-\mathrm{Bi} 1^{\mathrm{xxix}}$ & $3.6974(3)$ \\
\hline $\mathrm{Bi} 1-\mathrm{Bi}^{\mathrm{xiv}}$ & $3.6974(3)$ & $\mathrm{Bi} 2-\mathrm{Bi} 1^{\mathrm{xxx}}$ & $3.6974(3)$ \\
\hline $\mathrm{Bi} 1-\mathrm{Bi} 2^{\mathrm{xvi}}$ & $3.6974(3)$ & $\mathrm{Bi} 2-\mathrm{Bi}^{\mathrm{xxxi}}$ & $3.6974(3)$ \\
\hline $\mathrm{Bi} 1-\mathrm{Bi} 2^{\mathrm{xvii}}$ & $3.6974(3)$ & $\mathrm{Bi} 2-\mathrm{Bi} 1^{\mathrm{xxxii}}$ & $3.6974(3)$ \\
\hline $\mathrm{Bi} 1-\mathrm{Bi} 2^{\mathrm{xiii}}$ & $3.6974(3)$ & $\mathrm{Bi} 2-\mathrm{Bi}^{\mathrm{xii}}$ & $3.7310(4)$ \\
\hline $\mathrm{Bi} 1-\mathrm{Bi} 2^{\mathrm{xviii}}$ & $3.7309(4)$ & Ti1-Bi1 $1^{\text {xxii }}$ & $4.9242(16)$ \\
\hline $\mathrm{Bi} 1-\mathrm{Bi} 2^{\mathrm{xii}}$ & $3.7309(4)$ & Ti1-Bi1 $1^{x x i i i}$ & $4.9242(16)$ \\
\hline $\mathrm{Ti1} 1^{\mathrm{i}}-\mathrm{Bi} 2-\mathrm{Bi}^{\mathrm{xxx}}$ & $100.12(2)$ & $\mathrm{Bi} 2^{\mathrm{xiv}}-\mathrm{Bi} 1-\mathrm{Ti} 1^{\mathrm{xxiv}}$ & $36.352(10)$ \\
\hline $\mathrm{Bi} 1^{\mathrm{xxix}}-\mathrm{Bi} 2-\mathrm{Bi} 1^{\mathrm{xii}}$ & $100.174(6)$ & $\mathrm{Bi} 2-\mathrm{Bi} 1-\mathrm{Ti} 1^{\mathrm{xxiv}}$ & $36.426(10)$ \\
\hline $\mathrm{Bi} 2^{\mathrm{xvi}}-\mathrm{Bi} 1-\mathrm{Bi} 2^{\mathrm{xviii}}$ & $100.175(5)$ & $\mathrm{Bi} 1^{\mathrm{v}}-\mathrm{Ti} 1-\mathrm{Bi}^{\mathrm{xxii}}$ & $36.48(2)$ \\
\hline
\end{tabular}




\begin{tabular}{|c|c|c|c|}
\hline $\mathrm{Bi} 1^{\mathrm{xii}}-\mathrm{Bi} 1-\mathrm{Ti} 1^{\mathrm{xxii}}$ & $100.237(12)$ & $\mathrm{Bi} 1^{\mathrm{x}}-\mathrm{Bi} 1-\mathrm{Ti1}^{\mathrm{xxiv}}$ & $36.775(14)$ \\
\hline $\mathrm{Bi} 1^{\mathrm{xii}}-\mathrm{Bi} 1-\mathrm{Bi} 2^{\mathrm{xiii}}$ & $101.056(6)$ & $\mathrm{Bi} 2^{\mathrm{xiv}}-\mathrm{Bi} 1-\mathrm{Ti} 1^{\mathrm{xxii}}$ & $37.501(10)$ \\
\hline $\mathrm{Bi} 1^{\mathrm{xi}}-\mathrm{Bi} 1-\mathrm{Bi} 2^{\mathrm{xvii}}$ & $101.056(7)$ & $\mathrm{Bi} 2^{\mathrm{iii}}-\mathrm{Ti} 1-\mathrm{Bi}^{\mathrm{xxii}}$ & $48.51(2)$ \\
\hline $\mathrm{Bi} 2^{\mathrm{xvi}}-\mathrm{Bi} 1-\mathrm{Bi} 2^{\mathrm{xvii}}$ & $102.459(10)$ & $\mathrm{Bi} 1^{\mathrm{xxix}}-\mathrm{Bi} 2-\mathrm{Bi} 1^{\mathrm{xxx}}$ & $49.109(12)$ \\
\hline $\mathrm{Bi} 1^{\mathrm{xxix}}-\mathrm{Bi} 2-\mathrm{Bi} 1^{\mathrm{xxxii}}$ & $102.460(10)$ & Ti1 ${ }^{\mathrm{vi}}-\mathrm{Bi} 1-\mathrm{Bi} 2^{\mathrm{xix}}$ & $49.72(3)$ \\
\hline $\mathrm{Bi} 1^{\mathrm{xx}}-\mathrm{Bi} 2-\mathrm{Bi} 1^{\mathrm{xxxi}}$ & $102.460(11)$ & $\mathrm{Ti} 1^{\mathrm{xx}}-\mathrm{Bi} 1-\mathrm{Bi} 2^{\mathrm{xviii}}$ & $50.23(3)$ \\
\hline $\mathrm{Bi} 2^{\mathrm{xii}}-\mathrm{Bi} 2-\mathrm{Bi} 1^{\mathrm{xii}}$ & $102.596(8)$ & $\mathrm{Ti} 1^{\mathrm{vii}}-\mathrm{Bi} 1-\mathrm{Bi} 2^{\mathrm{xvii}}$ & $50.37(3)$ \\
\hline $\mathrm{Bi} 2^{\mathrm{xv}}-\mathrm{Bi} 2-\mathrm{Bi} 1^{\mathrm{xii}}$ & $103.120(6)$ & $\mathrm{Ti} 11^{\mathrm{i}}-\mathrm{Bi} 1-\mathrm{Bi} 2^{\mathrm{xviii}}$ & $51.27(3)$ \\
\hline $\mathrm{Ti} 1^{\mathrm{xi}}-\mathrm{Bi} 1-\mathrm{Bi} 1^{\mathrm{xi}}$ & $103.83(2)$ & $\mathrm{Ti} 1^{\mathrm{xi}}-\mathrm{Bi} 1-\mathrm{Bi} 2^{\mathrm{xiv}}$ & $51.93(3)$ \\
\hline $\mathrm{Bi} 1^{\mathrm{xii}}-\mathrm{Bi} 1-\mathrm{Bi} 2^{\mathrm{xvii}}$ & $103.916(6)$ & $\mathrm{Ti} 1^{\mathrm{i}}-\mathrm{Bi} 2-\mathrm{Bi} 1^{\mathrm{xii}}$ & $52.331(19)$ \\
\hline $\mathrm{Bi} 2^{\mathrm{xiv}}-\mathrm{Bi} 1-\mathrm{Bi} 2^{\mathrm{xix}}$ & $104.912(8)$ & $\mathrm{Ti}^{\mathrm{xxvii}}-\mathrm{Bi} 2-\mathrm{Bi} 1^{\mathrm{xxix}}$ & $52.441(7)$ \\
\hline $\mathrm{Ti} 1^{\mathrm{ix}}-\mathrm{Ti} 1-\mathrm{Bi} 1^{\mathrm{xxii}}$ & $105.95(7)$ & 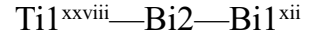 & $53.148(19)$ \\
\hline $\mathrm{Ti} 1^{\mathrm{xiv}}-\mathrm{Bi} 2-\mathrm{Bi} 2^{\mathrm{xiii}}$ & $106.077(14)$ & $\mathrm{Bi} 2^{\mathrm{xviii}}-\mathrm{Bi} 1-\mathrm{Bi} 2^{\mathrm{xii}}$ & $53.241(9)$ \\
\hline $\mathrm{Ti} 1^{\mathrm{i}}-\mathrm{Bi} 1-\mathrm{Bi} 1^{\mathrm{xi}}$ & $106.29(3)$ & $\mathrm{Bi} 2^{\mathrm{xvi}}-\mathrm{Bi} 1-\mathrm{Bi} 2^{\mathrm{xix}}$ & $53.310(6)$ \\
\hline $\mathrm{Bi}^{\mathrm{x}}-\mathrm{Bi} 1-\mathrm{Ti} 1^{\mathrm{vii}}$ & $106.58(4)$ & $\mathrm{Ti} 1^{\mathrm{xxi}}-\mathrm{Bi} 2-\mathrm{Bi} 1^{\mathrm{xxix}}$ & $53.901(6)$ \\
\hline 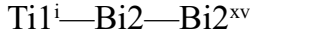 & $106.753(12)$ & $\mathrm{Ti} 1^{\mathrm{vii}}-\mathrm{Bi} 1-\mathrm{Bi} 1^{\mathrm{xi}}$ & $54.737(17)$ \\
\hline Ti1 $1^{x v i i}-B i 2-B i 2^{\text {xiii }}$ & $106.976(10)$ & $\mathrm{Ti} 1^{\mathrm{xuvi}}-\mathrm{Bi} 2-\mathrm{Bi} 2^{\mathrm{xv}}$ & $54.81(2)$ \\
\hline $\mathrm{Ti} 1^{\mathrm{xx}}-\mathrm{Bi} 1-\mathrm{Bi}^{\mathrm{x}}$ & $107.69(4)$ & Ti1 $1^{\mathrm{i}}-\mathrm{Bi} 2-\mathrm{Bi} 2^{\mathrm{xiii}}$ & $55.32(2)$ \\
\hline $\mathrm{Bi} 1^{\mathrm{vi}}-\mathrm{Ti} 1-\mathrm{Bi} 1^{\mathrm{xxii}}$ & $108.14(5)$ & $\mathrm{Bi} 1^{\mathrm{xxx}}-\mathrm{Bi} 2-\mathrm{Bi} 1^{\mathrm{xii}}$ & $55.502(6)$ \\
\hline Ti1 ${ }^{\text {xxvii_-Bi2-Ti1 }}{ }^{\text {xxviii }}$ & $109.61(4)$ & $\mathrm{Bi} 2^{\mathrm{xiv}}-\mathrm{Bi} 1-\mathrm{Bi} 2^{\mathrm{xvii}}$ & $55.865(12)$ \\
\hline Ti1 $1^{\mathrm{xx}}-\mathrm{Bi} 1-\mathrm{Ti} 1^{\mathrm{vii}}$ & $111.026(7)$ & $\mathrm{Ti}^{\mathrm{xx}}-\mathrm{Bi} 1-\mathrm{Bi}^{\mathrm{xi}}$ & $56.289(16)$ \\
\hline $\mathrm{Bi} 1^{\mathrm{xi}}-\mathrm{Bi} 1-\mathrm{Bi} 1^{\mathrm{xii}}$ & $117.32(2)$ & Ti1 ${ }^{\mathrm{xxi}}-\mathrm{Bi} 1-\mathrm{Ti}^{\mathrm{vii}}$ & $57.847(3)$ \\
\hline $\mathrm{Ti}^{\mathrm{ix}}-\mathrm{Ti1}-\mathrm{Bi}^{\mathrm{v}}$ & $117.43(3)$ & $\mathrm{Ti}^{\mathrm{viii}}-\mathrm{Ti} 1-\mathrm{Bi} 2^{\mathrm{iii}}$ & $59.07(5)$ \\
\hline $\mathrm{Ti} 1^{\mathrm{xxiv}}-\mathrm{Bi} 2-\mathrm{Bi} 1^{\mathrm{xii}}$ & $118.70(2)$ & Ti1 ${ }^{\text {xxiv }}-\mathrm{Bi} 2-\mathrm{Ti} 1^{\text {xxvii }}$ & $59.609(4)$ \\
\hline $\mathrm{Bi} 2^{\mathrm{xii}}-\mathrm{Bi} 2-\mathrm{Bi} 2^{\mathrm{xiv}}$ & $119.12(2)$ & $\mathrm{Ti} 1^{\mathrm{ix}}-\mathrm{Ti} 1-\mathrm{Bi} 1^{\mathrm{vii}}$ & $60.113(19)$ \\
\hline $\mathrm{Ti} 1^{\mathrm{ix}}-\mathrm{Ti} 1-\mathrm{Bi} 2^{\mathrm{iii}}$ & $119.48(9)$ & $\mathrm{Bi} 2^{\mathrm{ii}}-\mathrm{Ti} 1-\mathrm{Ti} 1^{\text {viii }}$ & $61.32(5)$ \\
\hline $\mathrm{Bi} 2^{\mathrm{xvi}}-\mathrm{Bi} 1-\mathrm{Ti} 1^{\mathrm{xxvi}}$ & $119.656(11)$ & Ti1 $1^{\text {xxiii-Bi1 }}-$ Ti1 $1^{\text {xxiv }}$ & $61.418(6)$ \\
\hline $\mathrm{Bi} 2^{\mathrm{xi}}-\mathrm{Bi} 1-\mathrm{Ti} 1^{\mathrm{xxii}}$ & $119.910(14)$ & $\mathrm{Bi} 1^{\mathrm{xii}}-\mathrm{Bi} 1-\mathrm{Bi} 2^{\mathrm{xviii}}$ & $61.757(12)$ \\
\hline $\mathrm{Bi} 2^{\mathrm{i}}-\mathrm{Ti} 1-\mathrm{Ti} 1^{\mathrm{viii}}$ & $120.13(9)$ & $\mathrm{Ti}^{\mathrm{viii}}-\mathrm{Ti} 1-\mathrm{Bi}^{\mathrm{v}}$ & $62.04(2)$ \\
\hline 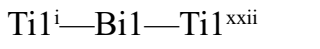 & $120.34(3)$ & $\mathrm{Bi} 2^{\mathrm{xv}}-\mathrm{Bi} 2-\mathrm{Bi} 1^{\mathrm{xxix}}$ & $62.067(6)$ \\
\hline Bi1 ${ }^{i}-\mathrm{Ti} 1-B i 1^{\mathrm{xxii}}$ & $120.34(3)$ & $\mathrm{Bi} 2^{\mathrm{xv}}-\mathrm{Bi} 2-\mathrm{Bi} 1^{\mathrm{xxx}}$ & $62.067(6)$ \\
\hline Ti1 $1^{\text {viii-Ti1-Bil }}{ }^{\text {vii }}$ & $120.39(3)$ & $\mathrm{Bi} 1^{\mathrm{xi}}-\mathrm{Bi} 1-\mathrm{Bi}^{\mathrm{xix}}$ & $62.132(6)$ \\
\hline $\mathrm{Bi2} 2^{\mathrm{ii}}-\mathrm{Ti} 1-\mathrm{Bi}^{\mathrm{iii}}$ & $120.391(4)$ & $\mathrm{Bi} 1^{\mathrm{xii}}-\mathrm{Bi} 1-\mathrm{Bi} 2^{\mathrm{xiv}}$ & $62.742(7)$ \\
\hline $\mathrm{Bi} 2^{\mathrm{xii}}-\mathrm{Bi} 2-\mathrm{Bi} 2^{\mathrm{xv}}$ & $120.438(12)$ & $\mathrm{Bi} 1^{\mathrm{xi}}-\mathrm{Bi} 1-\mathrm{Bi} 2^{\mathrm{xviii}}$ & $62.826(12)$ \\
\hline $\mathrm{Bi} 1^{\mathrm{x}}-\mathrm{Bi} 1-\mathrm{Bi} 1^{\mathrm{xi}}$ & $121.338(11)$ & $\mathrm{Bi} 2^{\mathrm{xiv}}-\mathrm{Bi} 2-\mathrm{Bi} 1^{\mathrm{xii}}$ & $63.380(4)$ \\
\hline Ti1 $1^{\mathrm{xx}}-\mathrm{Bi} 1-\mathrm{Ti} 1^{\mathrm{xxiv}}$ & $121.44(3)$ & $\mathrm{Bi} 2^{\mathrm{xiv}}-\mathrm{Bi} 2-\mathrm{Bi} 1^{\mathrm{xxix}}$ & $64.221(7)$ \\
\hline $\mathrm{Ti1}^{\mathrm{vii}}-\mathrm{Bi} 1-\mathrm{Ti}^{\mathrm{xxiv}}$ & $121.95(3)$ & $\mathrm{Bi} 2^{\mathrm{xiii}}-\mathrm{Bi} 2-\mathrm{Bi} 1^{\mathrm{xxxi}}$ & $64.221(7)$ \\
\hline $\mathrm{Bi} 2-\mathrm{Bi} 1-\mathrm{Ti} 1^{\mathrm{xxvi}}$ & $122.061(9)$ & $\mathrm{Bi} 1^{\mathrm{x}}-\mathrm{Bi} 1-\mathrm{Bi} 2^{\mathrm{xiv}}$ & $65.444(6)$ \\
\hline $\mathrm{Bi} 1^{\mathrm{vi}}-\mathrm{Ti} 1-\mathrm{Bi} 1^{\mathrm{vii}}$ & $122.154(4)$ & $\mathrm{Bi} 1^{\mathrm{x}}-\mathrm{Bi} 1-\mathrm{Bi} 2^{\mathrm{xix}}$ & $65.843(6)$ \\
\hline $\mathrm{Bi} 1^{\mathrm{xxix}}-\mathrm{Bi} 2-\mathrm{Bi} 1^{\mathrm{xxxi}}$ & $124.135(12)$ & $\mathrm{Bi} 2^{\mathrm{xix}}-\mathrm{Bi} 1-\mathrm{Ti} 1^{\mathrm{xxvi}}$ & $67.048(12)$ \\
\hline $\mathrm{Bi} 2^{\mathrm{xiv}}-\mathrm{Bi} 1-\mathrm{Bi} 2^{\mathrm{xviii}}$ & $124.499(6)$ & Ti1 $1^{\mathrm{xxv}}-\mathrm{Bi} 1-\mathrm{Ti} 1^{\mathrm{xxvi}}$ & $68.10(3)$ \\
\hline $\mathrm{Bi} 2^{\mathrm{xviii}}-\mathrm{Bi} 1-\mathrm{Bi} 2^{\mathrm{xix}}$ & $124.958(7)$ & $\mathrm{Bi} 2^{\mathrm{xvii}}-\mathrm{Bi} 1-\mathrm{Ti} 1^{\mathrm{xxvi}}$ & $68.52(2)$ \\
\hline Ti1 $1^{\mathrm{xxii}}-\mathrm{Bi} 1-\mathrm{Ti} 1^{\mathrm{xxvi}}$ & $129.41(3)$ & Bi1 $1^{v}-$ Til-Bi1 ${ }^{\text {vii }}$ & $68.975(7)$ \\
\hline $\mathrm{Bi} 1^{\mathrm{xii}}-\mathrm{Bi} 1-\mathrm{Ti}^{\mathrm{xxvi}}$ & $129.73(2)$ & Ti1 $1^{\mathrm{xxiii}}-\mathrm{Bi} 1-\mathrm{Ti} 1^{\mathrm{xxvi}}$ & $69.162(10)$ \\
\hline Ti1 ${ }^{\mathrm{xxiv}}-\mathrm{Bi} 1-\mathrm{Ti} 1^{\mathrm{xxvi}}$ & $130.440(11)$ & $\mathrm{Bi} 2^{\mathrm{i}}-\mathrm{Ti} 1-\mathrm{Bi} 2^{\mathrm{ii}}$ & $69.36(4)$ \\
\hline $\mathrm{Bi} 2^{\mathrm{xiv}}-\mathrm{Bi} 1-\mathrm{Bi} 2^{\mathrm{xvi}}$ & $130.889(12)$ & $\mathrm{Bi} 2^{\mathrm{xiii}}-\mathrm{Bi} 1-\mathrm{Ti} 1^{\mathrm{xxvi}}$ & $69.407(12)$ \\
\hline $\mathrm{Bi} 2^{\mathrm{xix}}-\mathrm{Bi} 1-\mathrm{Bi} 2$ & 131.685 (13) & $\mathrm{Bi} 2^{\mathrm{xiii}}-\mathrm{Bi} 1-\mathrm{Ti} 1^{\mathrm{xxiv}}$ & $69.516(7)$ \\
\hline $\mathrm{Bi} 1^{\mathrm{xi}}-\mathrm{Bi} 1-\mathrm{Ti} 1^{\mathrm{xxi}}$ & $131.728(9)$ & $\mathrm{Bi} 2^{i i i}-\mathrm{Ti} 1-\mathrm{Bi}^{2 \mathrm{iv}}$ & $70.39(4)$ \\
\hline
\end{tabular}




$$
\begin{aligned}
& \text { Bi2 }{ }^{\text {xviii }}-\mathrm{Bi} 1-\mathrm{Ti1} 1^{\mathrm{xxiv}} \\
& \mathrm{Bi} 2^{\mathrm{iii}}-\mathrm{Ti} 1-\mathrm{Bi}^{\mathrm{vii}} \\
& \text { Bi2-Ti1-Bi1 }{ }^{\mathrm{i}} \\
& \text { Bi2 }{ }^{x i i}-B i 1-T i 1^{x x i v} \\
& \text { Ti1 }{ }^{\text {xxi }}-\text { Bi1-Ti1 }{ }^{\text {xxvi }} \\
& \text { Bi1 }{ }^{\mathrm{xxxi}}-\mathrm{Bi} 2-\mathrm{Bi} 1^{\mathrm{xii}} \\
& \mathrm{Bi} 2^{\mathrm{xvii}}-\mathrm{Bi} 1-\mathrm{Bi} 2^{\mathrm{xvii}} \\
& \text { Ti1 } 1^{\mathrm{xx}}-\mathrm{Bi1}-\mathrm{Ti1} 1^{\mathrm{xxi}} \\
& \text { Bi1 }{ }^{v}-\mathrm{Ti} 1-\mathrm{Bi}^{\mathrm{vi}} \\
& \text { Ti1 }{ }^{\mathrm{xx}}-\mathrm{Bi} 1-\mathrm{Ti}^{\mathrm{xxi}} \\
& \text { Ti1 - Bi2-Ti1 }{ }^{\text {xiv }} \\
& \text { Ti1 }{ }^{\text {vii-Bi1-Ti1 }}{ }^{\mathrm{i}} \\
& \text { Bi1 } 1^{\text {vii_-Ti1-Bi1 }}{ }^{\mathrm{i}} \\
& \text { Ti1 }- \text { Bi2-Ti1 }{ }^{\text {xxvii }} \\
& \mathrm{Bi} 2^{\mathrm{i}}-\mathrm{Ti} 1-\mathrm{Bi} 2^{\mathrm{iii}} \\
& \text { Ti1 }-\mathrm{Bi} 2-\mathrm{Bi} 1^{\mathrm{xix}} \\
& \text { Ti1 }{ }^{\text {xxvii }}-\mathrm{Bi} 2-\mathrm{Bi} 1^{\mathrm{xii}} \\
& \text { Ti1 }{ }^{\mathrm{xx}}-\mathrm{Bi} 1-\mathrm{Bi} 2^{\mathrm{xiv}} \\
& \text { Ti1 }- \text { Bi1-Bi2 }{ }^{\text {xvii }} \\
& \text { Ti1 }{ }^{i}-B i 1-B i 2^{x i x} \\
& \mathrm{Bi} 1^{\mathrm{xi}}-\mathrm{Bi} 1-\mathrm{Bi}^{\mathrm{xiv}} \\
& B i 1^{\text {xii }}-B i 1-B i 2^{\text {xix }} \\
& \mathrm{Bi} 1^{x}-\mathrm{Bi} 1-\mathrm{Bi}^{\text {xviii }} \\
& B 2^{x i i}-B i 2-B i 1^{x x i x} \\
& \text { Bi1 } 1^{x i}-B i 1-T i 1^{\text {xxiv }} \\
& \text { Bi2 }{ }^{\text {xviii-Bi1-Ti1 }}{ }^{x x i i} \\
& \text { Bi2 - Ti1-Bi1 }{ }^{\text {xii }} \\
& \text { Bi2 }{ }^{\text {xiv }}-\mathrm{Bi} 1-\mathrm{Ti}^{\mathrm{xxvi}}
\end{aligned}
$$

\begin{tabular}{|c|c|}
\hline $\mathrm{Bi} 2^{\mathrm{xix}}-\mathrm{Bi} 1-\mathrm{Ti}^{\mathrm{xxii}}$ & $70.622(7)$ \\
\hline $\mathrm{Bi} 2^{\mathrm{xvii}}-\mathrm{Bi} 1-\mathrm{Ti}^{\mathrm{xxiv}}$ & $71.591(8)$ \\
\hline Ti1 $1^{x x i}-B i 1-T i 1^{x x i i i}$ & $71.66(3)$ \\
\hline 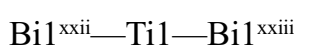 & $71.66(3)$ \\
\hline Ti1 $1^{\mathrm{xxi}}-\mathrm{Bi} 1-\mathrm{Ti} 1^{\mathrm{xxii}}$ & $71.85(5)$ \\
\hline Ti1 $1^{\text {viii_-Ti1-Bi1 }}{ }^{x x i i}$ & $72.76(6)$ \\
\hline Ti1 $1^{x x i v}-B i 1-T i 1^{x x v}$ & $73.55(3)$ \\
\hline $\mathrm{Bi} 2^{\mathrm{iv}}-\mathrm{Ti} 1-\mathrm{Bi}^{\mathrm{vii}}$ & $75.584(18)$ \\
\hline 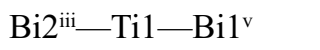 & $75.62(3)$ \\
\hline $\mathrm{Bi}^{2}-\mathrm{T} i 1-\mathrm{Bi} 1^{\mathrm{vii}}$ & 75.73 \\
\hline $\mathrm{Bi} 2^{\mathrm{ii}}-\mathrm{Ti} 1-\mathrm{Bi} 1^{\mathrm{vii}}$ & $77.12(3)$ \\
\hline 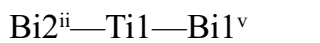 & $77.437(16)$ \\
\hline 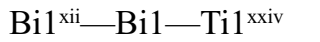 & $84.563(17)$ \\
\hline Ti1 $1^{\mathrm{vii}}-\mathrm{Bi} 1-\mathrm{Ti} 1^{\mathrm{xxvi}}$ & $85.661(13)$ \\
\hline Ti1 $1^{\mathrm{L}}-\mathrm{Bi} 1-\mathrm{Ti} 1^{\mathrm{xxiv}}$ & $85.87(4)$ \\
\hline 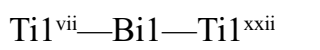 & $87.57(2)$ \\
\hline $\mathrm{Bi} 1^{\mathrm{vii}}-\mathrm{Ti} 1-\mathrm{Bi} 1^{\mathrm{xxii}}$ & $87.57(2)$ \\
\hline $\mathrm{Bi} 2^{\mathrm{xii}}-\mathrm{Bi} 1-\mathrm{Bi} 2^{\mathrm{xviii}}$ & $87.936(5)$ \\
\hline Ti1 ${ }^{\mathrm{xxi}}-\mathrm{Bi} 1-\mathrm{Ti} 1^{\mathrm{xxiv}}$ & $88.00(2)$ \\
\hline Ti1 $1^{\mathrm{i}}-\mathrm{Bi} 1-\mathrm{Ti1} 1^{\mathrm{xxvi}}$ & $88.706(13)$ \\
\hline $\mathrm{Bi} 1^{\mathrm{xxxi}}-\mathrm{Bi} 2-\mathrm{Bi} 1^{\mathrm{xii}}$ & $92.064(5)$ \\
\hline $\mathrm{Bi} 2^{\mathrm{xii}}-\mathrm{Bi} 1-\mathrm{Ti} 1^{\mathrm{xxvi}}$ & $93.35(2)$ \\
\hline 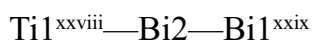 & $93.992(16)$ \\
\hline $\mathrm{Ti} 11^{\mathrm{i}}-\mathrm{Bi} 1-\mathrm{Bi}^{\mathrm{xiv}}$ & $95.236(16)$ \\
\hline Ti1 $1^{\mathrm{xxi}}-\mathrm{Bi} 2-\mathrm{Bi} 1^{\mathrm{xxx}}$ & $95.410(14)$ \\
\hline $\mathrm{Ti} 1^{\mathrm{xxi}}-\mathrm{Bi} 1-\mathrm{Bi} 2^{\mathrm{xviii}}$ & $95.54(4)$ \\
\hline $\mathrm{Ti} 1^{\mathrm{vii}}-\mathrm{Bi} 1-\mathrm{Bi} 2^{\mathrm{xviii}}$ & $96.63(4)$ \\
\hline $\mathrm{Bi} 2^{\mathrm{xvi}}-\mathrm{Bi} 1-\mathrm{Ti}^{\mathrm{xxii}}$ & $96.863(15)$ \\
\hline $\mathrm{Ti} 1^{\mathrm{xx}}-\mathrm{Bi} 1-\mathrm{Bi} 2^{\mathrm{xix}}$ & $97.142(14)$ \\
\hline $\mathrm{Bi} 2^{\mathrm{xvi}}-\mathrm{Bi} 1-\mathrm{Ti} 1^{\mathrm{xxiv}}$ & $98.027(15)$ \\
\hline $\mathrm{Ti} 1^{\mathrm{vii}}-\mathrm{Bi} 1-\mathrm{Bi} 2^{\mathrm{xiv}}$ & $98.391(15)$ \\
\hline $\mathrm{Bi} 2^{\mathrm{xix}}-\mathrm{Bi} 1-\mathrm{Ti} 1^{\mathrm{xiv}}$ & $98.570(15)$ \\
\hline $\mathrm{Bi} 2^{\mathrm{xii}}-\mathrm{Bi} 1-\mathrm{Bi} 2^{\mathrm{xix}}$ & $99.134(9)$ \\
\hline $\mathrm{Bi} 1^{x}-\mathrm{Bi} 1-\mathrm{Ti} 1^{\mathrm{xxvi}}$ & $99.91(2)$ \\
\hline
\end{tabular}

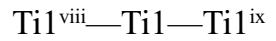

$$
\begin{aligned}
& \text { Ti1 }{ }^{\mathrm{xx}}-\mathrm{Bi} 1-\mathrm{Ti1}^{\mathrm{xxvi}} \\
& \text { Bi2 }{ }^{\text {xvi-Bi1-Ti1 }}{ }^{\text {xxi }} \\
& \text { Ti1 }{ }^{\text {xii }}-\text { Bi1-Ti1 }{ }^{\text {xxiv }} \\
& \text { Bi1 }{ }^{x i}-B i 1-T i 1^{x x v i} \\
& \text { Bi1 }{ }^{x}-B i 1-T i 1^{x x i i}
\end{aligned}
$$

Symmetry codes: (i) $-x,-y+1 / 2,-z+1 / 2$; (ii) $x+1 / 4, y-1 / 4,-z+1 / 2$; (iii) $-x+1 / 4,-y+3 / 4, z+1 / 2$; (iv) $x, y-1 / 2, z+1 / 2$; (v) $x+1 / 2, y, z+1 / 2$; (vi) $x-1 / 2, y$, $z+1 / 2$; (vii) $-x+1 / 2,-y,-z+1 / 2$; (viii) $-x+1 / 2,-y+1 / 2,-z+1$; (ix) $-x,-y,-z+1$; (x) $-x+1 / 4, y,-z+1 / 4$; (xi) $-x,-y,-z$; (xii) $-x+1 / 2,-y+1 / 2,-z$; (xiii) $-x-1 / 4,-y+3 / 4, z$; (xiv) $-x+3 / 4,-y+3 / 4, z$; (xv) $-x+1 / 4,-y+5 / 4, z$; (xvi) $x-1 / 2, y-1 / 2, z$; (xvii) $x+1 / 2, y-1 / 2, z$; (xviii) $x-1 / 4, y-1 / 4,-z$; (xix) $-x+1 / 4$, $-y+1 / 4, z$; (xx) $x-1 / 2, y, z-1 / 2$; (xxi) $x+1 / 2, y, z-1 / 2$; (xxii) $-x+3 / 4, y,-z+3 / 4$; (xxiii) $-x-1 / 4, y,-z+3 / 4$; (xxiv) $x+1 / 4,-y+1 / 2, z-1 / 4$; (xxv) $x-1 / 4,-y$, $z-1 / 4$; (xxvi) $-x-1 / 2,-y,-z+1 / 2$; (xxvii) $-x+1 / 4, y+1 / 2,-z+3 / 4$; (xxviii) $x, y+1 / 2, z-1 / 2$; (xxix) $-x+3 / 4, y+1 / 2,-z+1 / 4$; (xxx) $x+1 / 2, y+1 / 2, z$; (xxxi) $x-1 / 2, y+1 / 2, z$; (xxxii) $-x-1 / 4, y+1 / 2,-z+1 / 4$. 